\title{
NONHARMONIC FOURIER SERIES AND SPECTRAL THEORY
}

\author{
HAROLD E. BENZINGER
}

\begin{abstract}
We consider the problem of using functions $g_{n}(x):=\exp \left(i \lambda_{n} x\right)$ to form biorthogonal expansions in the spaces $L^{p}(-\pi, \pi)$, for various values of $p$. The work of Paley and Wiener and of Levinson considered conditions of the form $\left|\lambda_{n}-n\right| \leqslant$ $\Delta(p)$ which insure that $\left\{g_{n}\right\}$ is part of a biorthogonal system and the resulting biorthogonal expansions are pointwise equiconvergent with ordinary Fourier series. Norm convergence is obtained for $p=2$. In this paper, rather than imposing an explicit growth condition, we assume that $\left\{\lambda_{n}-n\right\}$ is a multiplier sequence on $L^{p}(-\pi, \pi)$. Conditions are given insuring that $\left\{g_{n}\right\}$ inherits both norm and pointwise convergence properties of ordinary Fourier series. Further, $\lambda_{n}$ and $g_{n}$ are shown to be the eigenvalues and eigenfunctions of an unbounded operator $\Lambda$ which is closely related to a differential operator, $i \Lambda$ generates a strongly continuous group and $-\Lambda^{2}$ generates a strongly continuous semigroup. Half-range expansions, involving $\cos \lambda_{n} x$ or $\sin \lambda_{n} x$ on $(0, \pi)$ are also shown to arise from linear operators which generate semigroups. Many of these results are obtained using the functional calculus for well-bounded operators.
\end{abstract}

1. Introduction. For $n$ an integer, let $\left\{\lambda_{n}\right\}$ be a sequence of pairwise distinct complex numbers. For $-\pi \leqslant x \leqslant \pi$ let

$$
g_{n}(x)=e^{i \lambda_{n} x}, \quad \varphi_{n}(x)=e^{i n x},
$$

and for integrable functions $f, g$ let

$$
(f, g)=\frac{1}{2 \pi} \int_{-\pi}^{\pi} f(x) \bar{g}(x) d x .
$$

Let $1 \leqslant p<\infty$ and $p^{-1}+q^{-1}=1$. For fixed $p$, assume there exists a sequence $\left\{h_{n}\right\}$ in $L^{q}\left(=L^{q}(-\pi, \pi)\right)$ such that

$$
\left(g_{n}, h_{m}\right)=\delta_{n m} .
$$

Then for $f$ in $L^{p}$, define the partial sum operator

$$
\mathscr{S}_{N}(x ; f)=\sum_{n=-N}^{N}\left(f, h_{n}\right) g_{n}(x) .
$$

The partial sum operator for ordinary Fourier series is

$$
S_{N}(x ; f)=\sum_{n=-N}^{N} \hat{f}_{n} \varphi_{n}(x), \quad \hat{f}_{n}=\left(f, \varphi_{n}\right) .
$$

Received by the editors September 1, 1985.

1980 Mathematics Subject Classification (1985 Revision). Primary 42C15, 42A45, 47A60; Secondary $34 \mathrm{~B} 25$.

(C)1987 American Mathematical Society $0002-9947 / 87 \$ 1.00+\$ .25$ per page 
The problem of nonharmonic Fourier series is to find conditions on $\left\{\lambda_{n}\right\}$ so that for some $p$, the dual sequence $\left\{h_{n}\right\}$ exists in $L^{q}$, and for all $f$ in $L^{p}$, the partial sum operators $\mathscr{S}_{N}(x ; f)$ have the same properties as the operators $S_{N}(x ; f)$, with respect to norm behavior, pointwise behavior, or both.

In this paper we shall consider these questions, subject to the basic assumption that the sequence $\left\{\delta_{n}\right\}$, defined by

$$
\delta_{n}=\lambda_{n}-n,
$$

is a multiplier sequence on $L^{p}$ for some fixed but arbitrary $p, 1 \leqslant p<\infty$. This means that there is a bounded linear operator $\mathscr{M}: L^{p} \rightarrow L^{p}$ such that for each $f$ in $L^{p}$,

$$
(\mathscr{M} f)_{n}=\delta_{n} \hat{f}_{n} .
$$

Another significant property of the sequences $\{n\}$ and $\left\{\varphi_{n}\right\}$ is that they contain the eigenvalues and eigenfunctions of the differential operator $\Lambda_{0}$ defined by

$$
\Lambda_{0} u=-i u^{\prime}, \quad\left(u^{\prime}=d u / d x\right),
$$

with domain $\mathscr{D}\left(\Lambda_{0}\right)$ consisting of all absolutely continuous functions $u$ such that $u^{\prime}$ is in $L^{p}$ and such that

$$
u(-\pi)=u(\pi)
$$

Thus

$$
\Lambda_{0} \varphi_{n}=n \varphi_{n} .
$$

For $p=2$ the operator $\Lambda_{0}$ is selfadjoint. For $1<p<\infty$ the spectral theory of $\Lambda_{0}$ is embodied in the statement that for some complex number $\lambda$ in the resolvent set of $\Lambda_{0}$, the resolvent operator $R\left(\lambda, \Lambda_{0}\right)$ is well-bounded. See [2] for the definition and applications to differential operators. We shall give conditions under which there exists a linear operator $\Lambda$ such that

$$
\Lambda g_{n}=\lambda_{n} g_{n},
$$

and such that the resolvent operator is well-bounded, $1<p<\infty$. This is then used to study the properties of half-range expansions, i.e., expansions on $L^{p}(0, \pi)$ (or on $\left.L^{p}(-\pi, 0)\right)$ using the sequence $\left\{\cos \lambda_{n} x\right\}$ or $\left\{\sin \lambda_{n} x\right\}$. In particular, we show that the operators associated with these expansions generate strongly continuous semigroups.

The study of nonharmonic Fourier series was initiated by Paley and Wiener [8] and by Levinson [7]. Paley and Wiener showed that for $p=2$ and $\lambda_{n}$ real, if $\left|\delta_{n}\right| \leqslant 1 / \pi^{2}$, then $\left\{h_{n}\right\}$ exists and for any $f$ in $L^{2}(-\pi, \pi)$, the partial sums $\mathscr{S}_{n}(x ; f)$ and $S_{n}(x ; f)$ have the same behavior with respect to pointwise convergence:

$$
\lim _{N \rightarrow \infty}\left[\mathscr{S}_{N}(x ; f)-S_{N}(x ; f)\right]=0,
$$

uniformly on each closed subinterval interior to $(-\pi, \pi)$. With respect to convergence in the norm of $L^{2}(-\pi, \pi)$, Paley and Wiener also showed that $\left\{g_{n}\right\}$ is a Riesz basis: there exists a bounded and invertible linear operator $A$ on $L^{2}$ such that

$$
A \varphi_{n}=g_{n},
$$

and thus $\left\{g_{n}\right\}$ has the same norm convergence properties in $L^{2}$ as does $\left\{\varphi_{n}\right\}$. 
The above result on pointwise convergence was generalized by Levinson, who showed that if $1<p \leqslant 2$ and if

$$
\left|\delta_{n}\right| \leqslant L<(p-1) / 2 p
$$

then $\left\{h_{n}\right\}$ exists and for any $f$ in $L^{p}(-\pi, \pi)$ the partial sums $\mathscr{S}_{N}(x ; f)$ and $S_{N}(x ; f)$ are uniformly equiconvergent on closed intervals interior to $(-\pi, \pi)$. Levinson did not give any results on the norm convergence of $\mathscr{S}_{N}$.

The question of norm convergence was considered by Pollard in [10]. There it was shown that for $1<p<\infty$, if $r=2 p /|2-p|$ and if $\left\{\delta_{n}\right\}$ is in $l^{r}$, with

$$
\left\|\left\{\delta_{n}\right\}\right\|_{r}<(\ln 2) / \pi,
$$

then $\left\{g_{n}\right\}$ is a basis for $L^{p}$ and there exists a bounded invertible operator $A: L^{p} \rightarrow L^{p}$ such that (1.13) holds. If $p=2$ then $r=\infty$ and (1.15) becomes

$$
\left|\delta_{n}\right| \leqslant L<(\ln 2) / \pi \text {. }
$$

This result for $p=2$ had been obtained earlier by Duffin and Eachus [4].

All of these conditions on $\left\{\delta_{n}\right\}$, whether for pointwise convergence, norm convergence, or both, impose a limitation on $\left\{\delta_{n}\right\}$ : in none of these conditions is $\left|\delta_{n}\right|$ allowed to be greater than $\frac{1}{4}$. Consider the example $\delta_{n}=\delta$ for all $n$, where $\delta$ is an arbitrary complex number. Then

$$
g_{n}(x)=e^{i \delta x} \varphi_{n}(x) .
$$

It is a simple matter to see that even if $\delta$ is selected so that none of the above conditions are satisfied, the resulting $\left\{g_{n}\right\}$ satisfies all of the conclusions of the above theorems, and in fact more is true: the pointwise equiconvergence theorem holds in the larger class $L^{\prime}(-\pi, \pi)$, and $\left\{g_{n}\right\}$ is the set of eigenfunctions of an unbounded linear operator which generates a strongly continuous bounded group of transformations on $L^{p}, 1<p<\infty$, and whose square generates a strongly continuous semigroup.

The conditions given by Paley and Wiener and by Pollard imply that $\left\{\delta_{n}\right\}$ is a multiplier sequence, and the same clearly holds for the above example. Thus the assumption that $\left\{\delta_{n}\right\}$ is a multiplier sequence contains all of the previous norm results, frees the theory from explicit growth conditions, and allows the association to each sequence $\left\{g_{n}\right\}$ of an unbounded linear operator whose spectral theory incorporates the norm properties of $\left\{g_{n}\right\}$. Further, if $\left\{\delta_{n}\right\}$ is a multiplier sequence and if $\left\{g_{n}\right\}$ is a basis for $L^{p}$ equivalent to $\left\{\varphi_{n}\right\}$, then pointwise equiconvergence is also obtained. Levinson's results are not included in this theory.

A survey of nonharmonic Fourier series is in [13] and other recent results on norm behavior can be found in $[14,15]$.

\section{Norm convergence.}

2.1. Definition. The sequences $\left\{g_{n}\right\},\left\{\varphi_{n}\right\}$ are equivalent in $L^{p}$ if there exists a bounded linear operator $A: L^{p} \rightarrow L^{p}$, with bounded inverse, such that

$$
A \varphi_{n}=g_{n} .
$$


Note that the definition applies for $p=1$, where $\left\{\varphi_{n}\right\}$ is not a basis. The invertibility of $A$ is sufficient for the existence of the dual sequence $\left\{h_{n}\right\}$ in $L^{q}$ :

$$
h_{n}=A^{-1 *} \varphi_{n} .
$$

2.4. LeMma. If $\left\{g_{n}\right\}$ and $\left\{\varphi_{n}\right\}$ are equivalent, then

$$
\mathscr{S}_{N}=A S_{N} A^{-1} \text {. }
$$

Proof. From (2.2) and (2.3) we have $\left(f, h_{n}\right) g_{n}=A\left(A^{-1} f, \varphi_{n}\right) \varphi_{n}$.

2.6. THEOREM. If $\left\{g_{n}\right\}$ is equivalent to $\left\{\varphi_{n}\right\}$ in $L^{p}, 1<p<\infty$, then

$$
\lim _{N \rightarrow \infty}\left\|\mathscr{S}_{N} f-f\right\|_{p}=0 .
$$

If $\left\{g_{n}\right\}$ is equivalent to $\left\{\varphi_{n}\right\}$ in $L^{1}$, then the arithmetic means of $\mathscr{S}_{N} f$ converge to $f$ in the norm of $L^{1}$.

Proof. We have $\mathscr{S}_{N}-I=A\left[S_{N}-I\right] A^{-1}$ and

$$
\frac{1}{N+1} \sum_{n=0}^{N} \mathscr{S}_{N}-I=A\left[\frac{1}{N+1} \sum_{n=0}^{N} S_{n}-I\right] A^{-1} \text {. }
$$

Thus $\mathscr{S}_{N}$ inherits the properties of $S_{N}$.

Let $X: L^{p} \rightarrow L^{p}$ be the linear operator defined by

$$
(X f)(x)=x f(x) \text {. }
$$

Note that $\|X\|=\pi$.

2.8. THEOREM. If $\left\{\delta_{n}\right\}$ is a multiplier sequence for some $L^{p}, 1 \leqslant p<\infty$, and if $A$ is the linear operator defined by

$$
A=\sum_{k=0}^{\infty} \frac{(i X)^{k} \mathscr{M}^{k}}{k !}
$$

then $A \varphi_{n}=g_{n}$. (It is not claimed that $A$ is invertible.)

Proof. If there exists an operator $A$ such that $A \varphi_{n}=g_{n}$, then for any trigonometric polynomial

$$
t(x)=\sum_{n=-N}^{N} \hat{t}_{n} \varphi_{n}(x)
$$

we must have

$$
A t=\sum_{n=-N}^{N} \hat{t}_{n} g_{n}
$$

Now $g_{n}(x)=\varphi_{n}(x) e^{i \delta_{n} x}$, so

$$
A t=\sum_{n=-N}^{N} \hat{t}_{n} \varphi_{n} \sum_{k=0}^{\infty} \frac{(i x)^{k} \delta_{n}^{k}}{k !}=\sum_{k=0}^{\infty} \frac{(i x)^{k}}{k !} \sum_{n=-N}^{N} \delta_{n}^{k} \hat{t}_{n} \varphi_{n} .
$$

Since $\mathscr{M}^{k} t=\sum_{-N}^{N} \delta_{n}^{k} \hat{t}_{n} \varphi_{n}$, we have

$$
A t=\sum_{k=0}^{\infty} \frac{(i X)^{k} \mathscr{M}^{k}}{k !} t, \quad \text { and } \quad\|A t\| \leqslant e^{\pi\|\mathscr{M}\|}\|t\| .
$$


Since the trigonometric polynomials are dense in $L^{p}$ for $1 \leqslant p<\infty$, the extension to all of $L^{p}$ of the operator defined by (2.10) is the operator defined in (2.9).

2.11. THEOREM. If for some $p, 1 \leqslant p<\infty$,

$$
\|\mathscr{M}\|_{p}<(\ln 2) / \pi
$$

then $\left\{g_{n}\right\}$ is equivalent to $\left\{\varphi_{n}\right\}$ in $L^{p}$.

Proof. It suffices to show that $\|A-I\|<1$. From (2.9),

$$
\|A-I\| \leqslant \sum_{k=1}^{\infty} \frac{\|X\|^{k}\|\mathscr{M}\|^{k}}{k !}=e^{\pi\|\mathscr{M}\|}-1 .
$$

Then (2.12) follows from the condition $e^{\pi\|\mathcal{M}\|}-1<1$.

This theorem contains the theorems of Duffin and Eachus and of Pollard. Using the Fredholm alternative to invert operators of the form $I-K$, where $K$ is compact, along with a representation of the dual sequence given by Levinson [7, Lemma 16.2], condition (1.15) of Pollard's theorem can be eliminated.

2.13. Theorem. Let $1<p<\infty, p \neq 2$, and let $r=2 p /|2-p|$. Then $\left\{g_{n}\right\}$ and $\left\{\varphi_{n}\right\}$ are equivalent if

(i) $\lambda_{n} \neq \lambda_{m}$ for $n \neq m$;

(ii) $\left\{\delta_{n}\right\}$ is in $l^{r}$.

The proof follows some preliminary material.

2.14. Lemma. Let $\left\{u_{n}\right\}$ be a sequence in a Banach space $\mathscr{B}$ and let $\left\{v_{n}\right\}$ be a sequence in a dual space $\mathscr{B}^{*}$ such that $\left(u_{n}, v_{m}\right)=\delta_{n m}$. Let $\left\{g_{n}\right\}$ be a sequence in $\mathscr{B}$ such that

(1) $g_{n}=u_{n}$ except for $n$ in a finite set $S$;

(2) $\operatorname{det}\left(\left(g_{n}, v_{m}\right)\right)_{n, m}$ in $S \neq 0$.

Then there exists a bounded, invertible operator $A: \mathscr{B} \rightarrow \mathscr{B}$ such that $A u_{n}=g_{n}$.

Proof. For $f$ in $\mathscr{B}$ define an operator $K$ by

$$
K f=\sum_{n \in S}\left(f, v_{n}\right)\left(u_{n}-g_{n}\right),
$$

and let $A=I-K$. Then $K$ is compact and $A u_{n}=g_{n}$ for all $n$. To show that $A$ is invertible it suffices to show (by the Fredholm alternative) that $A f=0$ implies $f=0$. We have $A f=0$ if and only if $f=K f$ :

$$
f=\sum_{n \in S}\left(f, v_{n}\right)\left(u_{n}-g_{n}\right) \text {. }
$$

Then for $m$ in $S$,

$$
\sum_{n \in S}\left(f, v_{n}\right)\left(g_{n}, v_{m}\right)=0 .
$$

From condition (2), $\left(f, v_{n}\right)=0$ for all $n$ in $S$, and from (2.15), $f=0$.

For Levinson's representation of $h_{n}$, we need the Fourier transform $\mathscr{F}$ and its inverse $\mathscr{F}^{-1}$ defined by

$$
(\mathscr{F} f)(\lambda)=\int_{-\infty}^{\infty} f(x) e^{-i \lambda x} d x \quad(f \text { defined on }(-\infty, \infty))
$$




$$
\left(\mathscr{F}^{-1} F\right)(x)=\frac{1}{2 \pi} \int_{-\infty}^{\infty} F(\lambda) e^{i \lambda x} d \lambda
$$

For the sequence $\left\{\lambda_{n}\right\}$, let

$$
G(\lambda)=\left(\lambda-\lambda_{0}\right) \prod_{n=1}^{\infty}\left(1-\frac{\lambda}{\lambda_{n}}\right)\left(1-\frac{\lambda}{\lambda_{-n}}\right) .
$$

Questions of convergence will be considered below. For $1<p<\infty$, let $p^{-1}+q^{-1}$ $=1, s=\min (p, q), s^{-1}+t^{-1}=1 . L^{p}$ refers to the interval $(-\pi, \pi)$ and $L^{p}(\mathbf{R})$ refers to $(-\infty, \infty)$.

2.19. TheOREM (LeVInSOn [7; pp. 48-58]). Let $1<p<\infty$. Assume

$$
\left|\delta_{n}\right| \leqslant L<(s-1) / 2 s .
$$

Then the infinite product (2.18) converges to an entire function $G(\lambda)$ such that if

$$
H_{n}(\lambda)=G(\lambda) /\left[\left(\lambda-\lambda_{n}\right) G^{\prime}\left(\lambda_{n}\right)\right],
$$

then

(i) $H_{n}$ is in $L^{s}(\mathbf{R})$ for $\lambda$ restricted to $\mathbf{R}$,

(ii) $\left(\mathscr{F}^{-1} H_{n}\right)(x)$ is in $L^{t}(\mathbf{R})$, and its support is contained in $(-\pi, \pi)$,

(iii) the dual sequence $\left\{h_{n}\right\}$ is given by

$$
\bar{h}_{n}(x)=2 \pi\left(\mathscr{F}^{-1} H_{n}\right)(x), \quad-\pi<x<\pi,
$$

and

$$
\frac{1}{2 \pi} \int_{-\pi}^{\pi} e^{i \lambda x} \bar{h}_{n}(x) d x=H_{n}(\lambda), \quad \lambda \in \mathbf{C} .
$$

2.24. REMARKS. Levinson's theorems are stated for $1<p \leqslant 2$, but using the containment relations for $L^{p}$ spaces on finite intervals, the above extension of the range of $p$ holds. Also, what is denoted by $h_{n}$ in Levinson's work is $2 \pi \bar{h}_{n}$ in our notation.

2.25. Lemma. For a finite set $S$ of indices, let $\left\{\lambda_{n}\right\},\left\{\mu_{n}\right\}, n \in S$, be two sets of complex numbers such that no two numbers are the same. Then

$$
M:=\operatorname{det}\left(\left(\lambda_{n}-\mu_{m}\right)^{-1}\right)_{n, m \in S} \neq 0 .
$$

Proof. Let $p(\lambda)=\Pi_{m \in S}\left(\lambda-\mu_{m}\right)$ and let $p_{i}(\lambda)=p(\lambda) /\left(\lambda-\mu_{i}\right)$. Then

$$
\frac{1}{\lambda_{n}-\mu_{m}}=\frac{p_{m}\left(\lambda_{n}\right)}{p\left(\lambda_{n}\right)}, \quad\left(p\left(\lambda_{n}\right) \neq 0\right) \text {. }
$$

Thus

$$
\left[\prod_{n=1}^{\infty} p\left(\lambda_{n}\right)\right] M=\operatorname{det}\left(p_{m}\left(\lambda_{n}\right)\right) .
$$

Now each $p_{m}$ is a polynomial of degree $|S|-1$, where $|S|$ is the cardinality of $S$, and all zeros of $p_{m}(\lambda)$ are accounted for by $\lambda=\mu_{i}$, where $i \in S, i \neq m$. Since $\lambda_{n} \neq \mu_{i}$, we have $M \neq 0$. 
Proof of Theorem 2.13. There exists a finite set $S$ of indices $n$ such that $\lambda_{n} \neq n$ for $n \in S,\left|\delta_{n}\right| \leqslant L<(s-1) / 2 s$, and

$$
\left(\sum_{n \notin S}\left|\delta_{n}\right|^{r}\right)^{1 / r}<(\ln 2) / \pi
$$

Let $\mu_{n}=m$ for $n \in S, \mu_{n}=\lambda_{n}$ for $n \notin S$, and let $u_{n}(x)=e^{i \mu_{n} x}$. Since $\left\{\mu_{n}-m\right\}$ satisfies Pollard's theorem (or Theorem 2.11), we see that $\left\{u_{n}\right\}$ is equivalent to $\left\{\varphi_{n}\right\}$. Let $\left\{v_{n}\right\}$ denote the dual sequence. Since $\left\{\mu_{m}-n\right\}$ also satisfies Levinson's condition (2.20), we have

$$
\frac{1}{2 \pi} \int_{-\pi}^{\pi} e^{i \lambda x} \bar{v}_{m}(x) d x=H_{m}(\lambda) .
$$

Since $\left\{g_{n}\right\}$ and $\left\{u_{n}\right\}$ differ only for $n \in S$, to show that $\left\{g_{n}\right\}$ and $\left\{u_{n}\right\}$ are equivalent it suffices to show that

$$
\operatorname{det}\left(\left(g_{n}, v_{m}\right)\right)_{n, m \in S} \neq 0 .
$$

Using (2.26), this becomes

$$
\operatorname{det}\left(H_{m}\left(\lambda_{n}\right)\right)_{n, m \in S} \neq 0
$$

Using (2.21), this becomes

$$
\left[\prod_{n \in S} \frac{G\left(\lambda_{n}\right)}{G^{\prime}\left(\mu_{n}\right)}\right]\left[\operatorname{det}\left(\left(\lambda_{n}-\mu_{m}\right)^{-1}\right)_{n, m \in S}\right] \neq 0 .
$$

Recall that $G(\lambda)$ is formed with zeros at $\left\{\mu_{m}\right\}$, so $G\left(\lambda_{n}\right) \neq 0$ for $n \in S$. Since the set $\left\{\lambda_{n}\right\}$ is disjoint from the set $\left\{\mu_{n}\right\}$ for $n \in S$, the determinant in (2.27) is not zero.

2.28 REMARK. The analogue of Theorem 2.13 for $p=2$ is that $\left|\delta_{n}\right| \leqslant L<(\ln 2) / \pi$ for $|n|$ sufficiently large, and, for the finitely many remaining $\lambda_{n}$ 's, that they are pairwise distinct.

For $p \neq 2$, Theorem 2.13 requires that $\delta_{n} \rightarrow 0$ as $|n| \rightarrow \infty$. Using the theory of well-bounded operators, a general class of multipliers can be given for which $\delta_{n} \rightarrow 0$ is not necessary. A special case will yield a proof of a theorem of Kadec [6]:

THEOREM (KADEC). Let $\left\{\delta_{n}\right\}$ be real and assume $\left|\delta_{n}\right| \leqslant L<\frac{1}{4}$. Then $\left\{g_{n}\right\}$ is a basis for $L^{2}$ equivalent to $\left\{\varphi_{n}\right\}$.

Some of the details of this theory are now presented.

2.29. Definition. An arc $C$ in the complex plane is admissible if it is simple, nonclosed and rectifiable:

Let $S$ denote the length of $C$ and let $\rho:[0, S] \rightarrow C$ denote the arc-length parameterization of $C$, with $b=\rho(S)$. A function $f: C \rightarrow \mathrm{C}$ is said to be absolutely continuous on $C$ if $f \circ \rho$ is absolutely continuous on $[0, S]$, and for such functions $f$, we define

$$
\||f|\|_{C}=|f(b)|+\int_{C}|d f / d z||d z| .
$$

2.31. Definition (RINGrose [12, p. 634]). An operator $T$ on a Banach space is well-bounded on $C$ if there exists a constant $K>0$ such that if $p(z)$ is any 
polynomial, then

$$
\|p(T)\| \leqslant K\|\mid p\|_{C} .
$$

2.33. TheOREM [12, p. 636]. If $T$ is well-bounded on $C$, then for each absolutely continuous function $f$ on $C$, there is a bounded linear operator $f(T)$ such that the mapping $f \rightarrow f(T)$ is a homomorphism of $A C(C)$ into the algebra of bounded linear operators, and

$$
\|f(T)\| \leqslant K\||f|\|_{C} .
$$

If the underlying Banach space is reflexive, then there exists a family of projections $\{E(\lambda): \lambda \in C\}$ a spectral family for $T$, which can be used to express $f(T)$ as a modified Riemann-Stieltjes integral [3, Chapter 17]. See also [2, Proposition 2.3], where we see that the constant $K$ of (2.34) can be chosen to be $\sup \{\|E(\lambda)\|: \lambda \in C\}$.

For $\Delta>0$, let

(2.35) $T_{\Delta}=\Delta T, \quad C_{\Delta}=\{\Delta z: z \in C\}, \quad E_{\Delta}(\lambda)=E(\lambda / \Delta), \quad \lambda \in C_{\Delta}$.

2.36. THEOREM. $T_{\Delta}$ is a well-bounded operator on $C_{\Delta}$ with spectral family $E_{\Delta}$, and for any function $f$ which is absolutely continuous on $C_{\Delta}$,

$$
\left\|f\left(T_{\Delta}\right)\right\| \leqslant K\||f|\|_{C_{\Delta}},
$$

where

$$
K=\sup \left\{E_{\Delta}(\lambda): \lambda \in C_{\Delta}\right\}=\sup \{E(\lambda): \lambda \in C\} .
$$

For the proof of this theorem, see [1] for a general discussion of functions of well-bounded operators. We emphasize that the constant $K$ for $T_{\Delta}$ in (2.37) is computable from the spectral family for $T$.

Multiplier transforms which are well-bounded have been studied by D. J. Ralph [11].

2.39. Definition. A real sequence $\left\{\delta_{n}\right\},-\infty<n<\infty$, is piecewise monotone if $\left\{\delta_{n}\right\}$ is monotone for $|n|$ sufficiently large. A complex sequence $\left\{\delta_{n}\right\}$ lying on an admissible arc $C$ is piecewise monotone if $\left\{\rho^{-1}\left(\delta_{n}\right)\right\}$ is piecewise monotone.

Note that the sense of monotonicity does not have to be the same for the two tails of $\left\{\delta_{n}\right\}$.

2.40. TheOREM [11, CoROllaRy 3.2.6]. If $\left\{\delta_{n}\right\}$ is a piecewise monotone sequence on an admissible arc $C$, then $\left\{\delta_{n}\right\}$ is a multiplier sequence for $L^{p}, 1<p<\infty$, and the associated multiplier transform $\mathscr{M}$ is well-bounded on $C$. Moreover, if $f$ is absolutely continuous on $C$, then for any $g$ in $L^{p}$

$$
(f(T) g)_{n}=f\left(\delta_{n}\right) \hat{g}_{n} .
$$

The proof of the next theorem depends upon the expression (due to Kadec [6]) of $1-e^{i \delta x}$ in the orthonormal system $\left\{1, \cos n x, \sin \left(n-\frac{1}{2}\right) x\right\}$ for $n \geqslant 1$ :

$$
\begin{aligned}
1-e^{i \delta x}= & \left(1-\frac{\sin \pi \delta}{\pi \delta}\right)+\frac{2}{\pi} \sum_{k=1}^{\infty} \frac{(-1)^{k} \delta \sin \pi \delta}{k^{2}-\delta^{2}} \cos k x \\
& +i \frac{2}{\pi} \sum_{k=1}^{\infty} \frac{(-1)^{k} \delta \cos \pi \delta}{\left(k-\frac{1}{2}\right)^{2}-\delta^{2}} \sin \left(k-\frac{1}{2}\right) x .
\end{aligned}
$$


2.43. THEOREM. Let $M$ be a well-bounded multiplier transform on some $L^{p}$, $1<p<\infty$, with multiplier sequence $\left\{\delta_{n}\right\}$. Then there exists $\Delta=\Delta(\mathscr{M}, p)>0$ such that if $\lambda_{n}=n+\Delta \delta_{n}$, then $\left\{g_{n}\right\}$ is a basis for $L^{p}$ equivalent to $\left\{\varphi_{n}\right\}$.

Proof. Let $f$ be a trigonometric polynomial in $L^{p}: f=\sum_{-N}^{N} \hat{f}_{n} \varphi_{n}$. For such $f$, $A f=\sum_{-N}^{N} \hat{f}_{n} g_{n}$ exists, and $B=I-A$ is defined:

$$
B f=\sum_{-N}^{N} \hat{f}_{n} \varphi_{n}\left[1-e^{i \Delta \delta_{n} x}\right] .
$$

Using (2.42) with $\delta=\Delta \delta_{n}$ and then interchanging the order of summation, we have

$$
\begin{aligned}
B f= & \sum_{n=-N}^{N}\left(1-\frac{\sin \pi \Delta \delta_{n}}{\pi \Delta \delta_{n}}\right) \hat{f}_{n} \varphi_{n} \\
& +\frac{2}{\pi} \sum_{k=1}^{\infty}(-1)^{k} \cos k x \sum_{n=-N}^{N} \frac{\Delta \delta_{n} \sin \pi \Delta \delta_{n}}{k^{2}-\left(\Delta \delta_{n}\right)^{2}} \hat{f}_{n} \varphi_{n} \\
& +\frac{i 2}{\pi} \sum_{k=1}^{\infty}(-1)^{k} \sin \left(k-\frac{1}{2}\right) x \sum_{n=-N}^{N} \frac{\Delta \delta_{n} \cos \pi \Delta \delta_{n}}{\left(k-\frac{1}{2}\right)^{2}-\left(\Delta \delta_{n}\right)^{2}} \hat{f}_{n} \varphi_{n} .
\end{aligned}
$$

Define functions

$$
\begin{gathered}
\alpha(\delta)=1-\frac{\sin \pi \delta}{\pi \delta}, \quad \beta_{k}(\delta)=\frac{\delta \sin \pi \delta}{k^{2}-\delta^{2}} \\
\gamma_{k}(\delta)=\frac{\delta \cos \pi \delta}{\left(k-\frac{1}{2}\right)^{2}-\delta^{2}}, \quad k=1,2, \ldots
\end{gathered}
$$

These functions are absolutely continuous on any admissible arc, and by Theorem 2.40, for any $f$ in $L^{p}$,

$$
\begin{gathered}
\alpha\left(\mathscr{M}_{\Delta}\right) f=\sum_{-\infty}^{\infty} \alpha\left(\Delta \delta_{n}\right) \hat{f}_{n} \varphi_{n}, \quad \beta_{k}\left(\mathscr{M}_{\Delta}\right) f=\sum_{-\infty}^{\infty} \beta_{k}\left(\Delta \delta_{n}\right) \hat{f}_{n} \varphi_{n}, \\
\gamma_{k}\left(\mathscr{M}_{\Delta}\right) f=\sum_{-\infty}^{\infty} \gamma_{k}\left(\Delta \delta_{n}\right) \hat{f}_{n} \varphi_{n} .
\end{gathered}
$$

Thus, using the density in $L^{p}$ of the trigonometric polynomials, we see that the operators $A, B$ have continuous extensions to all of $L^{p}$, and

$$
\begin{aligned}
B f= & \alpha\left(\mathscr{M}_{\Delta}\right) f+\frac{2}{\pi} \sum_{k=1}^{\infty}(-1)^{k} \cos k x \beta_{k}\left(\mathscr{M}_{\Delta}\right) f \\
& +\frac{2 i}{\pi} \sum_{k=1}^{\infty}(-1)^{k} \sin \left(k-\frac{1}{2}\right) x \gamma_{k}\left(\mathscr{M}_{\Delta}\right) f .
\end{aligned}
$$

Let $K>0$ be selected as in (2.38), where $E(\lambda)$ is the spectral family of $\mathscr{M}$. Using the triangle inequality,

$$
\|B f\| \leqslant\|f\| K\left\{\||\alpha|\|_{C_{\Delta}}+\frac{2}{\pi} \sum_{k=1}^{\infty}\left\|\left|\beta_{k}\right|\right\|_{C_{\Delta}}+\frac{2}{\pi} \sum_{k=1}^{\infty}\left\|\left|\gamma_{k}\right|\right\|_{C_{\Delta}}\right\} .
$$


Note that

$$
\begin{array}{ll}
\left|\beta_{k}(\delta)\right|=\mathcal{O}\left(\delta / k^{2}\right), & \text { uniformly as } \delta \rightarrow 0, k \rightarrow \infty, \\
\left|\beta_{k}^{\prime}(\delta)\right|=\mathcal{O}\left(\delta / k^{2}\right), & \text { also uniformly, }
\end{array}
$$

so that

$$
\left\|\left|\beta_{k}\right|\right\|_{C_{\Delta}}=\mathcal{O}\left(\Delta / k^{2}\right) \text { as } k \rightarrow \infty .
$$

Using similar estimates for $\alpha, \gamma_{k}$, we see that for $\Delta$ sufficiently small, $\|B\|<1$, and then $A$ is invertible.

If $\left\{\delta_{n}\right\}$ is real, then more precision in estimating $\left\|\left|\beta_{k}\right|\right\|$, etc., can be obtained. For $|\delta| \leqslant L \leqslant \frac{1}{4}$ we see that $\operatorname{var} \beta_{k}=2 \beta_{k}(L)$ so that

$$
\left\|\left|\beta_{k}\right|\right\|_{[-L, L]}=3 \beta_{k}(L)
$$

and similarly for $\alpha, \gamma_{k}$. Thus

$$
\|B f\| \leqslant\|f\| 3 K\left\{1-\frac{\sin \pi \Delta L}{\pi \Delta L}+\frac{2}{\pi} \sum_{k=1}^{\infty} \frac{\Delta L \sin \pi \Delta L}{k^{2}-(\Delta L)^{2}}+\frac{2}{\pi} \sum_{k=1}^{\infty} \frac{\Delta L \cos \pi \Delta L}{\left(k-\frac{1}{2}\right)^{2}-(\Delta L)^{2}}\right\} .
$$

Again using Kadec [6], we note that

$$
\begin{aligned}
\frac{\Delta 2 L}{\pi} \sum_{1}^{\infty} \frac{1}{k^{2}-(\Delta L)^{2}} & =\frac{1}{\Delta \pi L}-\cot \pi \Delta L, \\
\frac{2 \Delta L}{\pi} \sum_{1}^{\infty} \frac{1}{\left(k-\frac{1}{2}\right)^{2}-(\Delta L)^{2}} & =\tan \pi \Delta L,
\end{aligned}
$$

so

$$
\begin{gathered}
\|B f\| \leqslant\|f\| 3 K\left\{1-\frac{\sin \pi \Delta L}{\pi \Delta L}+\frac{\sin \pi \Delta L}{\pi \Delta L}-\cos \pi \Delta L+\sin \pi \Delta L\right\} \\
\|B\| \leqslant 3 K[1-\cos \pi \Delta L+\sin \pi \Delta L] .
\end{gathered}
$$

Then for $\Delta$ sufficiently small, $\|B\|<1$.

To say how small $\Delta$ should be, it is necessary to know $K$. Let $H$ denote the conjugate function mapping on $L^{p}$. For $1<p<\infty$, let $s=\min (p, q)$. Then [9]

$$
\|H\|_{p}=\tan (\pi / 2 r) .
$$

Using the representation of the spectral family of $\mathscr{M}[\mathbf{1 1}$, Theorem 3.2.4] we have:

2.46. LEMMA. If $\left\{\delta_{n}\right\}$ is real and piecewise monotone, and if $m$ is the number of intervals (of integers) on which $\left\{\delta_{n}\right\}$ is monotone, then

$$
K \leqslant m[1+\tan \pi / 2 r] .
$$

Kadec's theorem was based on Parseval's equality. A spectral-theoretic proof can be given, since $\mathscr{M}$ is then selfadjoint and $\left\|\beta_{k}(\mathscr{M})\right\|=\sup \left(\left|\beta_{k}\left(\delta_{n}\right)\right|\right)$, etc. 


\section{Pointwise convergence.}

3.1. TheOREM. Let $p$ be fixed, $1 \leqslant p<\infty$. Assume $\left\{\delta_{n}\right\}$ is a multiplier sequence in $L^{p}$ and that $\left\{g_{n}\right\},\left\{\varphi_{n}\right\}$ are equivalent. Then for each $f$ in $L^{p}$,

$$
\lim _{N \rightarrow \infty}\left[\mathscr{S}_{N}(x ; f)-S_{N}(x ; f)\right]=0
$$

uniformly on each interval $[-\pi+d, \pi-d], d>0$.

3.2. RemarK. Note that this theorem includes the case $p=1$, even though $\left\{\varphi_{n}\right\}$, $\left\{g_{n}\right\}$ are not bases in $L^{1}$. Theorem 3.1 contains as a special case a result of Duffin and Schaeffer [5, §4] for $L^{2}$.

Proof of Theorem 3.1. Since $\left\{g_{n}\right\},\left\{\varphi_{n}\right\}$ are equivalent, we have $\mathscr{S}_{N}=A S_{N} A^{-1}$. Using the expression (2.9) for $A$, we have

$$
\mathscr{S}_{N}=\sum_{k=0}^{\infty} X^{k} \frac{(i \mathscr{M})^{k}}{k !} S_{N} A^{-1},
$$

but since $\mathscr{M}$ and $S_{N}$ commute,

$$
\mathscr{S}_{N}=\sum_{k=0}^{\infty} X^{k} S_{N} \frac{(i \mathscr{M})^{k}}{k !} A^{-1} .
$$

Since $S_{N}=S_{N} A A^{-1}$, we have

$$
S_{N}=\sum_{k=0}^{\infty} S_{N} X^{k} \frac{(i \mathscr{M})^{k}}{k !} A^{-1},
$$

and then

$$
\mathscr{S}_{N} f-S_{N} f=\sum_{k=1}^{\infty}\left(X^{k} S_{N}-S_{N} X^{k}\right) \frac{(i \mathscr{M})^{k}}{k !} A^{-1} f .
$$

Let $D_{N}$ denote the Dirichlet kernel

$$
D_{N}(x-t)=\frac{\sin \left(N+\frac{1}{2}\right)(x-t)}{2 \sin ((x-t) / 2)}
$$

For any function $g$ in $L^{p}$,

$$
\left(X^{k} S_{N}-S_{N} X^{k}\right) g(x)=\int_{-\pi}^{\pi} D_{N}(x-t)\left(x^{k}-t^{k}\right) g(t) d t
$$

where

$$
\begin{aligned}
& D_{N}(x-t)\left(x^{k}-t^{k}\right) \\
& =\sin \left(N+\frac{1}{2}\right)(x-t) \frac{x-t}{2 \sin (x-t) / 2}\left[x^{k-1}+x^{k-2} t+\cdots+t^{k-1}\right] .
\end{aligned}
$$

Given $d>0$, there exists $K=K(d)>0$ such that if $|x| \leqslant \pi-d$, then

$$
\left|D_{N}(x-t)\left(x^{k}-t^{k}\right)\right| \leqslant K k \pi^{k}, \quad|x| \leqslant \pi-d,|t| \leqslant \pi .
$$

Thus

$$
\left|\left(X^{k} S_{N}-S_{N} X^{k}\right) g(x)\right| \leqslant 2 \pi K k \pi^{k}\|g\| .
$$


Let $\varepsilon>0$ be given. Then there exists $J=J(\varepsilon, f)$ such that

$$
\left|\sum_{k=J}^{\infty}\left(X^{k} S_{N}-S_{N} X^{k}\right) \frac{(i \mathscr{M})^{k}}{k !} A^{-1} f\right|<\frac{\varepsilon}{2}
$$

for all $N,|x| \leqslant \pi-d$. For the finitely many remaining terms, it is easily seen that the Riemann-Lebesgue lemma holds uniformly in $x,|x| \leqslant \pi-d$, so for $N$ sufficiently large,

$$
\left|\sum_{k=1}^{J-1}\left(X^{k} S_{N}-S_{N} X^{k}\right) \frac{(i \mathscr{M})^{k}}{k !} A^{-1} f\right|<\frac{\varepsilon}{2} .
$$

4. Eigenfunction expansions. In this section we assume $\left\{\delta_{n}\right\}$ is a multiplier sequence for $L^{p}$, for some $p, 1 \leqslant p<\infty$, and that the corresponding $\left\{g_{n}\right\}$ is equivalent to $\left\{\varphi_{n}\right\}$. Let $\Lambda_{0}$ be the differential operator defined in (1.8), (1.9), and let $\Lambda$ be defined by

$$
\Lambda=A\left(\Lambda_{0}+\mathscr{M}\right) A^{-1}, \quad \mathscr{D}(\Lambda)=A \mathscr{D}\left(\Lambda_{0}\right) .
$$

4.2. ThEOREM. $\Lambda$ is a closed, densely defined operator on $L^{p}$,

$$
\Lambda g_{n}=\lambda_{n} g_{n},
$$

and $i \Lambda$ is the infinitesimal generator of the uniformly bounded, strongly continuous group

$$
U(t)=A U_{0}(t) e^{i \mathscr{M} t} A^{-1}, \quad t \in \mathbf{R},
$$

where $U_{0}(t)$ is the translation group generated by $i \Lambda_{0}$.

Proof. This is a direct consequence of (4.1), noting that $\Lambda_{0}$ and $\mathscr{M}$ commute.

For the further study of $\Lambda$, let $1<p<\infty$. Then

$$
(\lambda I-\Lambda)^{-1} f=\sum_{-\infty}^{\infty}\left(\lambda-\lambda_{n}\right)^{-1}\left(f, h_{n}\right) g_{n}, \quad f \in L^{p} .
$$

Since $\left\{\left(\lambda-\lambda_{n}\right)^{-1}\right\}$ is in $l^{r}$ for all $r, 1<r<\infty$, it follows that $\left\{\left(\lambda-\lambda_{n}\right)^{-1}\right\}$ is a multiplier sequence in $L^{p}$ for $1<p<\infty$. For $(\lambda I-\Lambda)^{-1}$ to be well-bounded, it suffices to have $\left\{\left(\lambda-\lambda_{n}\right)^{-1}\right\}$ piecewise monotone. If $\left\{\delta_{n}\right\}$ is real, this is the case if $\left|\delta_{n}\right| \leqslant L<1 / 2$.

4.6. LEMMA. Let $\delta_{n}=\alpha_{n}+i \beta_{n}$ where

$$
\left|\alpha_{n}\right|<L<\frac{1}{2}, \quad \beta_{n}=\mathcal{O}(1)
$$

for $n$ sufficiently large. Let $\lambda$ be a real number distinct from the $\lambda_{n}$. Then $\left\{\left(\lambda_{n}-\lambda\right)^{-1}\right\}$ lies on an admissible arc $C$ and is piecewise monotone.

Proof. If suffices to show that $\left\{\operatorname{Re}\left(\lambda_{n}-\lambda\right)^{-1}\right\}$ is piecewise monotone and $\left\{\operatorname{Im}\left(\lambda_{n}-\lambda\right)^{-1}\right\}$ is of bounded variation, since then the arc formed by joining successive points $\left(\lambda_{n}-\lambda\right)^{-1}$ with straight lines is admissible. A computation yields

$$
\operatorname{Re}\left(\lambda_{n}-\lambda\right)^{-1}=\frac{1}{n}-\frac{\alpha_{n}-\lambda}{n^{2}}+\frac{\gamma_{n}}{n^{3}}, \quad \gamma_{n}=\mathcal{O}(1),
$$


and then the difference of two successive ones is

$$
\frac{1}{n(n+1)}\left[1-\left(\alpha_{n}-\alpha_{n+1}\right)+\frac{\gamma_{n}}{n}\right] \text {. }
$$

For $|n|$ sufficiently large this is positive, since $\alpha_{n}-\alpha_{n+1}<1$. Clearly $\operatorname{Im}\left(\lambda_{n}-\lambda\right)^{-1}$ $=\mathcal{O}\left(n^{-2}\right)$, so this sequence is of bounded variation.

4.8. ThEOREM. If $\left\{\delta_{n}\right\}$ satisfies (4.7), then $R(\lambda, \Lambda)$ is well-bounded.

Proof. By the above lemma, $\left\{\left(\lambda-\lambda_{n}\right)^{-1}\right\}$ satisfies the conditions of [11, Corollary 3.2.6] (see also Theorem 2.40), so $R\left(\lambda, \Lambda_{0}+\mathscr{M}\right)$ is well-bounded. Well-boundedness is preserved by similarity transforms.

We have $\Lambda^{2}=A\left(\Lambda_{0}+\mathscr{M}\right)^{2} A^{-1}$ with domain $\mathscr{D}\left(\Lambda_{0}^{2}\right)$.

4.9. THEOREM. If $\lambda$ does not coincide with any $\lambda_{n}^{2}$ and if (4.7) holds, then $R\left(\lambda, \Lambda^{2}\right)$ is well-bounded on $L^{p}, 1<p<\infty$.

The proof is similar to that for $\Lambda$.

4.10. Corollary. For $1<p<\infty,-\Lambda^{2}$ is the infinitesimal generator of a semigroup in $L^{p}$.

Proof. Since the admissible arc $C$ containing $\left\{\left(\lambda-\lambda_{n}^{2}\right)^{-1}\right\}$ enters the origin with bounded slope, the conditions of [2, Theorem 5.15] are satisfied. (See also [2, Lemma 5.48].)

5. Half-range expansions. Assuming the sequence $\left\{\lambda_{n}\right\}$ is odd:

$$
\lambda_{-n}=-\lambda_{n} \text {, }
$$

we consider expansions for $0<x<\pi$ (or for $-\pi<x<0$ ) in $\left\{\cos \lambda_{n} x\right\}, n \geqslant 0$ and in $\left\{\sin \lambda_{n} x\right\}, n \geqslant 1$. We give conditions assuring that these functions are eigenfunctions of linear operators which generate strongly continuous semigroups in $L^{p}(0, \pi)$.

We assume throughout this section that (5.1) holds and $\left\{g_{n}\right\},\left\{\varphi_{n}\right\}$ are equivalent in some space $L^{p}$.

5.2. LEMMA. $g_{-n}(x)=g_{n}(-x), h_{-n}(x)=h_{n}(-x)$.

Proof. Since $\left\{g_{n}\right\}$ is given explicitly, this is an immediate consequence of (5.1). For $h_{n}$, let $m$ be fixed and let $w(x)=h_{m}(-x)$. Then for all $n$, and using the above property of $g_{n}$, we have $\left(g_{n}, w-h_{-m}\right)=0$ all $n, m$. Since $\left\{g_{n}\right\}$ is complete we have $w=h_{-m}$.

For the remainder of this section we consider cosine expansions. Thus let

$$
\begin{gathered}
G_{n}(x)=\left[g_{n}(x)+g_{-n}(x)\right] / 2, \quad H_{n}(x)=\left[h_{n}(x)+h_{-n}(x)\right] / 2, \\
c_{n}(x)=\cos n x .
\end{gathered}
$$

Clearly

$$
G_{n}=A c_{n}, \quad H_{n}=A^{-1 *} c_{n} .
$$

For an even function $f$ on $[-\pi, \pi]$, let

$$
F(x)=f(x), \quad 0<x<\pi,
$$


and for two functions $u, v$ on $(0, \pi)$, let

$$
\langle u, v\rangle=\frac{2}{\pi} \int_{0}^{\pi} u(x) \bar{v}(x) d x
$$

5.7. LEMMA. If $f$ is an even function, then for $0<x<\pi$,

$$
\begin{gathered}
\left(f, h_{0}\right) g_{0}=\frac{1}{2}\left\langle F, H_{0}\right\rangle G_{0}, \\
\left(f, h_{n}\right) g_{n}+\left(f, h_{-n}\right) g_{-n}=\left\langle F, H_{n}\right\rangle G_{n}, \\
\sum_{-N}^{N}\left(f, h_{n}\right) g_{n}=\frac{1}{2}\left\langle F, H_{0}\right\rangle G_{0}+\sum_{1}^{N}\left\langle F, H_{n}\right\rangle G_{n}:=\mathscr{T}_{N}(x ; F) .
\end{gathered}
$$

Proof. Computational.

Let

$$
T_{N}(x ; F)=\frac{1}{2}\left\langle F, c_{0}\right\rangle c_{0}(x)+\sum_{1}^{N}\left\langle F, c_{n}\right\rangle c_{n}(x), \quad 0<x<\pi .
$$

5.11. THEOREM. If (5.1) holds and if $\left\{g_{n}\right\},\left\{\varphi_{n}\right\}$ are equivalent in $L^{p}$ for some $p$, $1 \leqslant p<\infty$, then for all $F$ in $L^{p}(0, \pi)$,

$$
\lim _{N \rightarrow \infty}\left[\mathscr{T}_{N}(x ; F)-T_{N}(x ; F)\right]=0,
$$

uniformly on $[0, \pi-d]$ for each $d>0$. If $1<p<\infty$, then

$$
\lim _{N \rightarrow \infty} \mathscr{T}_{N}(\cdot ; F)=F
$$

in the norm of $L^{p}(0, \pi)$.

Proof. These are direct consequences of the relations

$$
\mathscr{S}_{N}(x ; f)=\mathscr{T}_{N}(x ; F), \quad S_{N}(x ; f)=T_{N}(x ; F), \quad 0<x<\pi,
$$

and the analogous theorems for $\mathscr{S}_{N}, S_{N}$.

If $f$ is an even function in $\mathscr{D}\left(\Lambda^{2}\right)$, then for $0<x<\pi$,

$$
\Lambda^{2} f=\sum_{1}^{\infty} \lambda_{n}^{2}\left\langle F, H_{n}\right\rangle G_{n}:=\Gamma^{2} F,
$$

where $\mathscr{D}\left(\Gamma^{2}\right)$ consists of all $F$ in $L^{p}(0, \pi)$ such that the even extension to $[-\pi, \pi]$ is in $\mathscr{D}\left(\Lambda^{2}\right)$. For $\lambda \neq \lambda_{n}^{2}$, and for any polynomial $P$,

$$
\begin{aligned}
P\left(R\left(\lambda, \Gamma^{2}\right)\right) F & =\frac{1}{2} P\left(\lambda^{-1}\right)\left\langle F, H_{0}\right\rangle G_{0}+\sum_{1}^{\infty} P\left(\left(\lambda-\lambda_{n}^{2}\right)^{-1}\right)\left\langle F, H_{n}\right\rangle G_{n} \\
& =P\left(R\left(\lambda_{n} \Lambda^{2}\right)\right) f, \quad 0<x<\pi
\end{aligned}
$$

5.13. THEOREM. If $\left\{\delta_{n}\right\}$ satisfies (4.7), along with the other assumptions of this section, then for $1<p<\infty, R\left(\lambda, \Gamma^{2}\right)$ is well bounded and $-\Gamma^{2}$ generates a strongly continuous semigroup on $L^{p}(0, \pi)$.

Proof. Since for any function $F$ and its even extension $f$ we have

$$
\|f\|^{p}=2\|F\|^{p}
$$


from (5.12) and the well-boundedness of $R\left(\lambda, \Lambda^{2}\right)$,

$$
\begin{aligned}
\left\|P\left(R\left(\lambda, \Gamma^{2}\right)\right) F\right\| & =2^{-1 / p}\left\|P\left(R\left(\lambda, \Lambda^{2}\right)\right) f\right\| \\
& \leqslant 2^{-1 / p} K\||P|\|\|f\|=K\|\mid P\|\|\| F \|,
\end{aligned}
$$

where $\||P|\|$ is computed on the piecewise linear admissible arc containing $\left\{\left(\lambda-\lambda_{n}^{2}\right)^{-1}\right\}$. Thus $R\left(\lambda, \Gamma^{2}\right)$ is well-bounded and the proof of Corollary 4.10 applies.

\section{REFERENCES}

1. H. E. Benzinger, Functions of well-bounded operators, Proc. Amer. Math. Soc. 92 (1984), 75-80.

2. H. E. Benzinger, E. Berkson and T. A. Gillespie, Spectral families of projections, semigroups and differential operators, Trans. Amer. Math. Soc. 275 (1983), 431-475.

3. H. R. Dowson, Spectral theory of linear operators, London Math. Soc. Mono., No. 12, Academic Press, New York, 1978.

4. R. J. Duffin and J. J. Eachus, Some notes on an expansion theorem of Paley and Wiener, Bull. Amer. Math. Soc. 48 (1942), 850-855.

5. R. J. Duffin and A. C. Schaeffer, A class of nonharmonic Fourier series, Trans. Amer. Math. Soc. 72 (1952), 341-366.

6. M. I. Kadec, The exact value of the Paley-Wiener constant, Soviet Math. Dokl. 5 (1964), 559-561.

7. N. Levinson, Gap and density theorems, Amer. Math. Soc. Colloq. Publ., Vol. 26, Amer. Math. Soc., Providence, R. I., 1940.

8. R. E. A. C. Paley and N. Wiener, Fourier transforms in the complex domain, Amer. Math. Soc. Colloq. Publ., Vol. 19, Amer. Math. Soc., Providence, R. I., 1934.

9. S. K. Pichorides, On the best value of the constants in the theorems of M. Riesz, Zygmund and Kolmogorov, Studia Math. 44 (1972), 165-179.

10. H. Pollard, The mean convergence of non-harmonic series, Bull. Amer. Math. Soc. 50 (1944), 583-586.

11. D. J. Ralph, Semigroups of well-bounded operators and multipliers, Thesis, Univ. of Edinburgh, 1977.

12. J. R. Ringrose, On well-bounded operators. II, Proc. London Math. Soc. (3) 13 (1963), 613-638.

13. R. M. Young, An introduction to nonharmonic Fourier series, Academic Press, New York, 1980.

14. R. M. Redheffer, Completeness of sets of complex exponentials, Adv. in Math. 24 (1977), 1-62.

15. R. M. Redheffer and R. M. Young, Completeness and basis properties of complex exponentials, Trans. Amer. Math. Soc. 277 (1983), 93-111.

Department of Mathematics, University of Illinois, 1409 W. Green Street, Urbana, Illinois 61801 